\title{
"ME NO REPENT YET": ESTRATEGIAS PARA LA TRADUCCIÓN DEL INGLÉS JAMAICANO EN BREVE HISTORIA DE SIETE ASESINATOS DE MARLON JAMES
}

\author{
Miguel Sanz Jiménez, Universidad Complutense de Madrid \\ Email: miguelsanz@ucm.es
}

\begin{abstract}
Resumen: Breve historia de siete asesinatos, de Marlon James, es una novela polifónica que refleja la variedad lingüística de sus protagonistas y narradores. En particular, las desviaciones de la norma estándar que supone el inglés jamaicano se marcan mediante la técnica denominada eye dialect. En este trabajo se estudian los rasgos de esta variedad que figuran en la novela, las distintas estrategias para traducir el dialecto en textos literarios y las prácticas editoriales respecto a este problema para después analizar el caso concreto de la versión española de la novela de James.
\end{abstract}

Palabras clave: Inglés jamaicano, Marlon James, Novela contemporánea, Traducción del dialecto, Traducción literaria, Variedad lingüística.

Title in English: "Me no repent yet": Strategies for translating Jamaican English in A Brief History of Seven Killings, by Marlon James.

Abstract: A Brief History of Seven Killings, by Marlon James, is a polyphonic novel that depicts the linguistic varieties spoken by its main characters and narrators. Particularly, it uses eye dialect to portray the deviations from the standard norm that Jamaican English represents. This paper studies the features of this variety that come up in the novel, as well as different strategies for translating dialect in literary texts, and publishing companies' policies regarding this issue. Ultimately, the Spanish version of James's novel will be analyzed according to these strategies.

Keywords: Contemporary Narrative, Jamaican English, Linguistic Varieties, Literary Translation, Marlon James, Translation of Dialect.

\section{INTRODUCCIÓN}

Este trabajo se enmarca en el campo de los Estudios Descriptivos de Traducción, que analizan las traducciones como productos concretos de las normas y convenciones de la cultura de la lengua meta. Si se comparan los textos de la lengua origen con sus respectivas traducciones, se observan las normas que rigen el proceso traductor, los criterios editoriales y cómo la cultura meta construye la imagen de la cultura fuente por medio de la traducción.

La novela objeto de estudio es Breve historia de siete asesinatos, de Marlon James. Una vez se haya tenido en cuenta el contexto de su publicación, se analizarán sus particularidades lingüísticas y los escollos de traducción que presenta. En concreto, se observará cómo se representa en la obra el inglés jamaicano y el patois o criollo jamaicano que hablan algunos 
de sus múltiples narradores. Dado que el lenguaje de los personajes indica que pertenecen a un colectivo específico y subraya las divisiones sociales, las novelas escritas en una variedad no estándar de la lengua origen, como la de James, presentan una dificultad añadida para la traducción. Por este motivo, se repasará cómo diversos traductólogos abordan la cuestión de la traducción de la variedad linguiística y qué estrategias proponen al respecto, se examinarán algunas prácticas editoriales con relación a esta cuestión y, finalmente, se comprobará, mediante un análisis cualitativo de los fragmentos seleccionados de la novela de James y sus correspondientes traducciones al castellano, qué estrategias se emplearon en el caso de la citada obra y del problema traductológico que plantea.

\section{BREVE HISTORIA DE SIETE ASESINATOS, DE MARLON JAMES}

\subsection{Contexto y publicación de la novela}

Breve historia de siete asesinatos (cuyo título original es A Brief History of Seven Killings) es la tercera novela de Marlon James, escritor jamaicano afincado en Estados Unidos, donde enseña literatura y escritura creativa en Macalester College, en Minnesota. Su primera novela, John Crow's Devil (2005), narra el conflicto religioso que se desarrolla en el pueblo jamaicano Gibbeah en 1957 y recuerda al lenguaje de escritores norteamericanos contemporáneos como Toni Morrison y Cormac McCarthy. En 2009 la siguió The Book of Night Women, la historia de una plantación de azúcar en la Jamaica de finales del siglo XVIII que, al igual que la novela con la que debutó, está inédita en castellano.

En 2014, James publicó Breve historia de siete asesinatos, que arranca con el fallido intento de asesinato del cantante de reggae Bob Marley en 1976, antes del concierto para promover la paz en Jamaica. La novela sigue las vidas de los asesinos, que pertenecen a la Storm Posse, la banda del gánster Papa-Lo que se funda en el gueto ficticio de Copenhagen City y expande su negocio de narcotráfico hasta Nueva York en la década de los noventa, cuando llega a enfrentarse al cártel de Medellín. Además, recoge los testimonios de periodistas y espías de la CIA cuyas tramas se cruzan con las que protagonizan los mentados criminales. Se basa, en parte, en la historia real de la Shower Posse, del barrio Tivoli Gardens, y se trata de una novela compleja e intrincada de quince narradores distintos, todos ellos partícipes de la trama. Por su variedad de tonos, que abarcan del cómico al surrealista, el sentido del absurdo y las referencias constantes a la cultura popular, se la ha comprado con La broma infinita, de David Foster Wallace (Lazar 2014); mientras que la violencia gráfica y la versión alternativa de historia reciente que describe la novela recuerdan a la saga negrocriminal El cuarteto de Los Ángeles, de James Ellroy (Blincoe 2015). Mientras trabajaba en esta obra, James se preguntó si sería capaz de escribir al otro sin caer en el exotismo de los blancos que escriben personajes negros (Harvey 2015), por lo que mezcló voces y dialectos dispares que comprenden del inglés jamaicano al estadounidense e incluyen pasajes escritos en español, como sucede en varios episodios que protagoniza Josey Wales, un joven y ambicioso gánster que sabe desenvolverse en distintos registros y lenguas. Después de que se publicara en 2014, Breve historia de siete asesinatos cosechó los elogios de la crítica y ganó en 
premio Man Booker en 2015. En España, la traducción al castellano de Javier Calvo y Wendy Guerra se publicó en abril de 2016, un mes después que la versión catalana de Ramon Monton i Lara ${ }^{1}$.

\subsection{La variedad lingüística en la novela}

Gracias a la mezcla de tonos que se mencionaba en el apartado anterior, James juega con la estructura de la novela y fractura la narración con voces múltiples (Pardo 2016). Es decir, se trata de un caso de heteroglosia en novela, el fenómeno que Bakhtin define como "la diversidad de hablas sociales (a veces incluso de lenguas) y la diversidad de voces individuales organizadas artísticamente ${ }^{2 "}$ (1981:262). En particular, para reflejar el idiolecto particular de los protagonistas, Breve historia de siete asesinatos mezcla varios dialectos del inglés, que abarcan del jamaicano al estadounidense estándar. Se entiende por dialecto, según los lingüistas Crystal e Ivić (2010), "una variedad regional del lenguaje caracterizada por determinados rasgos léxicos, morfológicos, gramaticales y fonéticos [...] incluso por las peculiaridades que presenta un individuo concreto a la hora de expresarse". En otras palabras, se consideran dialectales las "variedades de la lengua geográficas, socioeconómicas, estilísticas y étnicas que difieren de la norma estándar” (Määttä 2004: 320). En la novela objeto de estudio, estas variedades se reflejan mediante la técnica denominada eye dialect, que el estudioso Zanger define como el intento de reflejar, en la página impresa, "por medio de una determinada ortografía o errores ortográficos, elisiones, apóstrofos, alteraciones sintácticas, signos, etc. el habla de un grupo étnico, regional o racial determinado "3" (1966: 40). Este empleo de variantes ortográficas para representar dialectos que se alejan del estándar es una convención ortotipográfica y no una precisa reproducción de la variedad lingüística por escrito (Walpole 1974: 196). La función de este dialecto literario depende de las convenciones y los estereotipos que se relacionen con sus rasgos en la cultura origen, pues puede ser objeto de burla o una marca de identidad, de forma que los personajes que lo hablan quedan marcados mediante el mencionado eye dialect.

En especial, en Breve historia de siete asesinatos se recurre a esta técnica para reflejar en la página impresa el patois jamaicano, una variedad vernácula del inglés cuya pronunciación, léxico y sintaxis difieren de la norma estándar. En la monografía que dedica a esta variedad lingüística, Bailey apunta que los dialectos criollos, en general, tienen menos prestigio social que la lengua estándar, que se impone como modelo, y explica que los hablantes del patois jamaicano son, en su mayoría, descendientes de los esclavos africanos que llevaron a la isla durante los siglos XVII, XVIII y a principios del XIX (1966: 2). Esta misma autora resume las principales diferencias entre el inglés estándar y el criollo jamaicano, que son la no concordancia de sujetos y verbos, la no flexión de las formas verbales en pasado (a diferencia de los participios que terminan en -ed en inglés estándar), la falta de voz pasiva en esta variedad jamaicana, la omisión de los verbos de cópula que introducen los adjetivos que califican a los sujetos y el uso del pronombre personal de tercera persona singular him

1 Cfr. James, M. 2016. Una breu historià de set assassinats. Trad. R. Monton i LaRA. Alzira: Bromera.

2 La traducción de las citas directas de la bibliografía en lengua inglesa es obra del autor de este trabajo, a menos que se especifique lo contrario.

3 Traducción de Rica Peromingo y Braga Riera (2015: 123). 
tanto para sujetos femeninos como masculinos ${ }^{4}$ (1966: 146). Gibson coincide con estas observaciones y apunta que, en el jamaicano, se suele utilizar el adverbio just al hablar del pasado, la preposición fi para expresar finalidad y el adverbio de frecuencia aawez (always) junto a verbos sin flexión para el presente (1988: 199-200).

Algunos de estos rasgos se pueden apreciar en los siguientes fragmentos de la novela, en los que el dialecto criollo que emplean el pueblo llano de la isla y los asesinos protagonistas queda retratado mediante el mencionado eye dialect y contrasta con los pasajes en dialecto estadounidense y en español. El primer fragmento corresponde a una advertencia de PapaLo, el líder de la Storm Posse durante la década de los setenta. En él se puede observar el uso de apóstrofos para sugerir una pronunciación no estándar, el pronombre personal me en sustitución del habitual $I$ y la ausencia de la $-s$ para la tercera persona del singular en los verbos en presente:

Me warn him y'know, my magnanimous gentlemens. Long time I drop warnings that other people close, friend and enemy was going get him in a whole heap o' trouble. Every one of we know at least one, do not it? Them kinda man who just stay a certain way? Always have a notion but never come up with a single idea. Always working plenty of scheme but never have a plan. That was certain people. Here is my friend the biggest superstar in the world and yet him have some of the smallest mind to come out of the ghetto as friend. Me not going name who but I warn the singer. I say, You have some people right close to you who going do nothing but take you down, you hear me? Me tired to say that to him. Sick and tired. But him just laugh that laugh, that laugh that swallow the room. That laugh that sounded like he already have a plan (James 2015: 23).

A continuación, estos dos pasajes ilustran la habilidad para cambiar de código de Josey Wales, uno de los pistoleros de la banda de Papa-Lo que acaba por destronarlo como líder. Su pericia lingüística resulta clave en el ascenso social que emprende a lo largo de la novela y en ambos fragmentos se observa cómo sabe hablar español y bajar el registro en inglés para despistar a sus interlocutores. Nótese que el texto original resalta en cursiva los pasajes en español:

I do not tell him that I about to set up a man in Miami and one in New York. I do not tell him that yo tengo suficiente español para conocer que eres la más gran broma en Sudamérica. I chat to him bad like some Bush naigger and ask dumb question like, So everybody in America have gun? What kinda bullet American fire? Why you do not transfer Dirty Harry to the Jamaica branch? Hee hee hee (James 2015: 44).

- Ha. My mistake. Pero todo es un error en este país de mierda.

- Not half a mistake as the stupid country you're coming from.

- ¿Por Dios, hablas español?

I nod yes.

-CIA hombre, he knows you think?

I nod no (James 2015: 166).

4 Para un estudio exhaustivo en este campo, véase el trabajo DurrLEMAn-Tame, S. 2008. The Syntax of Jamaican Creole: A Cartographic Perspective. Amsterdam / Philadelphia: John Benjamins. 
El contraste entre las variedades lingüísticas que recoge la novela de James también sale a relucir en la conversación que mantiene Alex Pierce, el periodista de la revista Rolling Stone (que se basa en la figura real de Cameron Crowe), con los guardias que custodian la casa de Bob Marley, a quien viene a visitar. Los malentendidos que despiertan los referentes culturales a los que alude Pierce propician una situación, cuando menos, cómica:

- Bombocloth. Look here, Taffie, Jesus is risen.

-What? But me no repent yet.

The white man didn't seem to get the joke. I stepped out of the way, maybe making too much of a show of it.

- Hey buddy, Alex Pierce from Rolling Stone.

- Wait deh now, tight jeans Jesus, Jehovah know say you lie? Two man from Rolling Stone come here already, one name Keith and one named Mick and none of them look like you.

- But them all resemble still, Eddie.

- True that. True that.

-I'm from Rolling Stone magazine. We spoke on the phone.

- You never talk to me on no phone.

-I mean, someone from in the office. His secretary or something I do not know. I'm from the magazine? From the U.S.? We cover everybody from Led Zeppelin to Elton John. I do not understand, the secretary said come December 3 at six p.m. when he's on rehearsal break and here I am.

-Bossman, me do not name sexetary.

-But-

- Look, we get strict orders. Nobody in or out except family and band.

- Oh. Why does everybody have an automatic weapon? You guys police? You do not look like the security guard from last time I was here.

- None of your damn business, you want step off now.

-Eddie, the man still bothering you at the gate?

- Him say him magazine is "bout Lesbian and Elton John.

- No, Led Zeppelin and-

- Tell him to move off.

- How about me making it easy for you (James 2015: 49-50).

La ausencia de cópulas, el empleo de verbos en presente sin flexión, la falta de verbos auxiliares en las formas continuas y el uso de la preposición $f i$ para expresar finalidad se aprecian en el episodio de brutalidad policial en el que se ve envuelto Demus, uno de los matones de Papa-Lo:

The police, six I count, say one of you is a nasty rapist who rape church women when they coming back from praising the Lord. And since you is all lying nasty ghetto boy me not even going ask the guilty perpetrator to step forward. We do not know what to do, because if any one of we get called the rapist the police going shoot him before he reach the jail. So the first police-man who talk all the time say, But we know how fi catch you. The whole of you drop to the ground now! We confused so we look around and me look at the soap bubbles popping one by one and exposing me business. The policeman fire two shot in the air and say drop-a-ground now! So we drop. [...] Now listen what me want you all fi do, he say. Me want all of you to fuck the ground good [...] Me say fi fuck the dirt, the policeman say (James 2015: 53). 
"Me no repent yet": Estrategias para la traducción...

Como muestran los ejemplos arriba expuestos, la variedad lingüística se emplea como elemento caracterizador de la novela de James. Surge, pues, el problema de cómo trasladar este juego de voces y sus implicaciones a la versión castellana del texto. En el siguiente apartado se examinará la posibilidad de traducir las variedades dialectales en narrativa y se prestará atención a distintas posturas traductológicas y prácticas editoriales con relación a este problema concreto.

\section{ESTRATEGIAS PARA LA TRADUCCIÓN DE LA VARIEDAD LINGÜÍSTICA}

\subsection{Consideraciones traductológicas}

Una vez se ha ilustrado con ejemplos el uso del dialecto jamaicano como elemento caracterizador en Breve historia de siete asesinatos, en este apartado se planteará si es conveniente reflejar estas desviaciones de la norma lingüística del texto origen en la lengua meta y qué estrategias se pueden seguir con tal fin.

Son varios los traductólogos que se muestran a favor de traducir este tipo de marcas, caso de López García, quien apunta que "dejar de traducir esos efectos expresivos de la lengua de origen priva al lector de una información cardinal para comprender la obra que se lee", por lo que recomienda "hacer llegar al lector la peculiar desviación expresiva de la norma que ofrece el dialecto" (1991: 93). A continuación, aconseja trasladar las connotaciones que se asocian a una variedad en particular y "explicar por qué razones [el traductor] ha elegido un determinado método de traducción” en un ejercicio de coherencia en la lengua de llegada (1991: 94-95). En esta misma línea se posiciona Rabadán, que sostiene que, si el mantenimiento del sentido depende de los rasgos dialectales, se debe elegir en la lengua meta un dialecto que pueda evocar las mismas connotaciones que el original y cumpla unas funciones de caracterización geográfica y social similares (1991: 79-109). Hatim y Mason profundizan en este campo y abordan el problema de a qué dialecto de la lengua meta se recurre en la traducción, puesto que se pierden las connotaciones del texto de origen si se traduce por la norma culta o estándar de la lengua de llegada. Estos dos autores subrayan la función sociocultural y las implicaciones políticas e ideológicas de los dialectos sociales y geográficos, ya que son consecuencia de la estratificación social de la comunidad lingüística. Asimismo, insisten en la necesidad de traducir estas variedades para respetar la carga social del original, si bien apuntan que no hay por qué elegir una variedad regional en concreto, sino que se puede optar por modificar el estándar, por ejemplo variando la gramática y el léxico en la lengua meta (1995: 55-64).

Siguiendo las observaciones de Hatim y Mason, Tello Fons (2012: 137-158) afirma que los traductores han de contemplar la posibilidad de verter la variedad lingüística y elegir entre una serie de opciones según la función que desempeñe el dialecto en el texto de origen. Esta traductóloga distingue dos funciones distintas del dialecto en una obra literaria, que son la función mimética, que retrata la realidad del habla de una comunidad lingüística determinada; y la función simbólica, que abarca oposiciones entre personajes y la ideología que proyecta un dialecto concreto. Independientemente de la función que desempeñe en el texto fuente, Tello Fons plantea cuatro estrategias para traducir la variedad lingüística. En primer lugar, se puede 
optar por un texto con marcas que transgredan la norma de la lengua de llegada en sus niveles ortográfico, gramatical o léxico, o bien por un texto sin marcas, es decir, estandarizado según las convenciones de la norma lingüística. En segundo lugar, se puede elegir entre traducir a un dialecto concreto de la lengua meta para domesticar el texto, y optar por una configuración artificial que combine rasgos de varios dialectos sin recordar a ninguno en particular. Estas dos últimas estrategias son las que Paradela López (2014) identifica, respectivamente, con el anatopismo, la traducción por dialectos reales de la lengua de llegada, y con el atopismo, es decir, la creación de dialectos literarios que no se correspondan con los de ningún lugar concreto pero sí ayuden a que el lector perciba una diferencia en el habla.

En el capítulo que dedican a la traducción de las variedades dialectales, Rica Peromingo y Braga Riera tienen en cuenta estas consideraciones de Tello Fons para vincular el sociolecto a las variables de clase, edad, generación, sexo, nivel económico, profesión, origen rural o urbano e incluso raza y religión y apuntan que corresponde al traductor captar la función de su uso en la lengua fuente y buscar una solución para su recepción en la lengua meta. Asimismo, ambos estudiosos reconocen que no existen soluciones unívocas, ya que las decisiones traductoras dependerán del género textual, de la función del dialecto en la obra literaria, del papel de la literatura traducida en el polisistema de la cultura meta y de la verosimilitud que se quiera aportar al texto traducido (2015: 133). Tras estas consideraciones, Rica Peromingo y Braga Riera se basan en las observaciones de la mentada Tello Fons y de Perteghella ${ }^{5}$ a propósito de la traducción de textos dramáticos y recopilan seis estrategias para la transposición del dialecto literario, que son las siguientes (2015: 134-143): la compilación dialectal o mezcla de dialectos y modismos propios de la lengua de llegada, que respeta la ambientación del original; la traducción pseudodialectal, que recurre al lenguaje no estándar y a los rasgos idiomáticos de varios dialectos de la lengua meta para crear una variedad ficticia y difícil de identificar (el atopismo de Paradela López); la traducción dialectal paralela, que se corresponde con el denominado anatopismo y opta por traducir por otro dialecto propio de la lengua de llegada que tenga connotaciones similares a la variedad del texto origen; la localización dialectal, un caso de domesticación en el que se trasladan el dialecto, las referencias culturales y hasta la ambientación del texto fuente a la cultura meta; la estandarización o nivelación cuando se traduce a la lengua estándar y se elimina la variedad lingüística del texto fuente para favorecer la comprensión, si bien se pierden las connotaciones de partida; y, por último, la compensación, que "permite que algunas estructuras marcadas se traduzcan a la lengua estándar del texto final, a la vez que otras no dialectales se marcan en la traducción" (2015: 141).

Si bien, como se verá en el siguiente apartado, las prácticas editoriales revelan la preferencia por la estandarización de la variedad lingüística en el texto meta, en este apartado se ha observado que sí hay varios traductólogos que se posicionan a favor de seguir estas estrategias dado que "el traductor debe proceder con precaución siempre, pero no debe tener miedo de intentar producir versiones innovadoras de textos innovadores o que se desvían de las normas de los cánones" (D’Amore 2010: 41).

5 Cfr. Perteghella, M. 2002. "Language and Politics on Stage: Strategies for Translating Dialect and Slang with References to Shaw's Pygmalion and Bond's Saved". Translation Review 64: 45-53. 


\subsection{Prácticas editoriales}

En lo que concierne al problema de la traducción de la variedad lingüística, la realidad de la práctica editorial tiende a hacer caso omiso de las posibilidades que ofrecen las estrategias traductoras expuestas en la sección anterior. En su manual de traducción literaria, Landers advierte de que "ningún dialecto viaja bien en la traducción" (1999: 117) y manifiesta su preferencia por eliminar las marcas dialectales del texto original con el fin de facilitar la comprensión lectora en la lengua meta. No en vano, como se verá a continuación, la estandarización o nivelación parece ser la tendencia al tratar el problema de la variedad lingüística en traducción literaria, una preferencia respaldada "por la ley de normalización progresiva proveniente de los Estudios Descriptivos de Traducción” (Sánchez Galvis 2013: 141).

Con relación a esta práctica extendida, el lingüista y traductor Bernárdez Sanchís explica que las editoriales suelen modificar las partes de la obra original que no se adecúan a los criterios que establecen las políticas de la empresa, por lo que llevan a cabo un "planchado" del original (2012: 95-99). Así, en la traducción al castellano explican lo que no queda muy claro, armonizan la estructura y el vocabulario para que no se produzcan cambios de registro ni tampoco se transgreda la norma estándar con el fin de que el texto meta "sea accesible al mayor número posible de lectores" (2012: 111). En el ensayo El fantasma en el libro, Calvo coincide con estas reflexiones y apunta que al traductor, desde las editoriales, se le impide que reproduzca las hablas regionales y los dialectos, pues, en general, las convenciones editoriales no permiten que se juegue con la dicción de los personajes, las marcas de oralidad del texto fuente ni tampoco con los vulgarismos, que se suprimen en la traducción al español (2016: 129).

A propósito de esta práctica editorial, resulta ilustrativo el caso clásico de la traducción de Las aventuras de Huckleberry Finn, de Mark Twain. La novela presenta el problema de la traducción del inglés vernáculo de los personajes negros, como Jim, y también de los blancos (Julià Ballbè 1997: 196); si bien el escollo de la traducción de la variedad lingüística está presente en la propia nota introductoria, que advierte a los lectores del empleo de hasta siete dialectos diferentes en la novela. Las soluciones traductoras varían ${ }^{6}$ y, si bien predomina la estandarización de las desviaciones de la norma culta, a veces se añade una nota a pie de página e incluso se suprime la mencionada advertencia inicial, que se reproduce a continuación:

Explanatory.

In this book a number of dialects are used, to wit: the Missouri negro dialect; the extremest form of the backwoods Southwestern dialect; the ordinary "Pike County" dialect; and four modified varieties of this last. The shadings have not been done in a haphazard fashion, or by guesswork; but painstakingly, and with the trustworthy guidance and support of personal familiarity with

6 Para un estudio detallado acerca de la traducción de la variedad lingüística en Las aventuras de Huckleberry Finn, véase Rodríguez Herrera, J. M. 2014. "The Reverse Side of Mark Twain's Brocade: The Adventures of Huckleberry Finn and the translation of dialect". European Journal of English Studies 18, 3: 278-294. 
these several forms of speech.

I make this explanation for the reason that without it many readers would suppose that all these characters were trying to talk alike and not succeeding.

The Author (Twain 2001: 167).

En la práctica de la traducción literaria, no obstante, sí se dan notables excepciones a las imposiciones editoriales recién referidas. La traducción de Sáenz de la novela Llámalo sueño, de Henry Roth (1990), resulta paradigmática por cómo juega con la ortografía, de modo que los personajes, en su mayoría inmigrantes en la Nueva York de principios del siglo xx, aspiran las eses y sus elles se convierten en íes griegas con el fin de que, a ojos del lector, parezca que hablan un dialecto coherente. Con relación a su trabajo en esta novela, Sáenz matiza que "el problema de la traducción del dialecto sí tiene muchas soluciones, mas todas ellas resultan insatisfactorias" (2000).

Otra excepción notable a la estrategia de nivelación, y volviendo al inglés caribeño presente en Breve historia de siete asesinatos, es la traducción al castellano de La maravillosa vida breve de Óscar Wao que llevó a cabo la escritora cubana Achy Obejas (Calvo 2016: 146-148). La novela de Junot Díaz presenta varios narradores de origen dominicano que emigran a Estados Unidos y está escrita en un inglés que mimetiza el ritmo y la sintaxis del español, abundan los culturemas hispanos y las profusas notas a pie de página que dan pistas a los lectores acerca de las referencias culturales que pueblan la novela. Es más, Díaz omite los signos de apertura de las exclamaciones e interrogaciones y las tildes en los pasajes en español, añade diéresis a palabras que no tienen por qué llevarla, transcribe la pronunciación de algunos vocablos españoles y adapta ciertas estructuras propias de la lengua española al inglés, caso de "He'd say, No be a baby" (Díaz 2008a: 16). A la hora de afrontar semejantes escollos, Obejas (2012) cuenta que, tras recibir el encargo de traducir La maravillosa vida breve de Óscar Wao, se dedicó a leer periódicos dominicanos, escuchar su radio y leer a escritores de este país. Cuando terminaba de traducir tres o cuatro capítulos, se los enviaba a Díaz, quien, a su vez, se los mandaba a sus amigos dominicanos más cercanos, que le devolvían el manuscrito con notas y comentarios. El resultado de esta cadena de revisiones es un texto híbrido en el que el cambio de código y la variedad lingüística del original no siempre se mantienen en los mismos pasajes del texto meta (Jiménez Carra 2011: 166), puesto que Obejas emplea la estrategia de compensación para añadir expresiones en inglés en otros lugares del texto en castellano ("Le dijo: Do not be a baby"; Díaz 2008b: 27), corrige las tildes omitidas e invierte los juegos de palabras del original, como se ilustra en el siguiente ejemplo:

\begin{tabular}{|l|l|}
\hline \multicolumn{1}{|c|}{ Texto original } & \multicolumn{1}{c|}{ Versión española } \\
\hline $\begin{array}{l}\text { Listen, we'll let you go if you tell us } \\
\text { what fuego means in English. }\end{array}$ & $\begin{array}{l}\text { Mira, te soltamos si nos dices qué } \\
\text { significa fire. }\end{array}$ \\
$\begin{array}{l}\text { Fire, he blurted out, unable to help } \\
\text { himself (Díaz 2008a: 332). }\end{array}$ & $\begin{array}{l}\text { Fuego, soltó, incapaz de contenerse } \\
\text { (Díaz 2008b: 293). }\end{array}$ \\
\hline
\end{tabular}

Tabla 1. La compensación en La maravillosa vida breve de Óscar Wao. 
En definitiva, la traducción de Obejas compensa el inglés dominicano con una versión en castellano que recuerda a las construcciones inglesas (Jiménez Carra 2011: 176) y, así, prescinde de las convenciones editoriales y ortotipográficas de la lengua meta para transmitir la atmósfera y la experiencia de la mezcla de culturas de la novela de Díaz?

Una vez se han tenido en cuenta las observaciones traductológicas y las prácticas editoriales relacionadas con el problema de la traducción de la variedad lingüística en narrativa, en la siguiente sección se aplicarán estas observaciones al análisis cualitativo del caso concreto de la traducción al castellano de Breve historia de siete asesinatos, la novela de Marlon James objeto de estudio de este trabajo.

\section{LATRADUCCIÓN AL ESPAÑOLDE BREVE HISTORIA DE SIETE ASESINATOS}

Como se ha visto en el segundo apartado de este trabajo, la heteroglosia de la novela de James caracteriza las voces de los narradores, los separa y diferencia entre sí. Es más, la variedad lingüística presente en el texto original y, en particular, el reflejo del patois jamaicano por medio del eye dialect suponen un escollo para la traducción de la novela al castellano, si bien, como se ha observado en la tercera sección de este estudio, varios traductólogos reflexionan a propósito de las estrategias para la traducción del dialecto en los textos literarios. Precisamente, la nota sobre la traducción que abre el volumen en español de Breve historia de siete asesinatos aborda estas cuestiones y explica cómo los traductores afrontaron este escollo, como puede leerse en el siguiente fragmento:

No hay narrador externo, no hay tercera persona. La historia se despliega como un enorme juego de contrapuntos polifónicos donde cada individuo (cada testigo) exhibe su propia voz singular e intransferible. Unos habitan el territorio de un inglés ciertamente jamaicano, pero más o menos canónico. Otros no salen del dialecto criollo (el patois) usado por el pueblo llano de la isla. Varios oscilan entre esas regiones o circulan por la frontera que las separa. Tres hablan un estadounidense genérico. ¿Cómo puede reflejarse esa variedad en la traducción? Si cada jerga "vulgar" está indeleblemente marcada por su tiempo y su espacio, ¿cómo podemos trasladarla a las volubles geografías de otro idioma? Todas las soluciones a ese viejo (e intratable) problema son, en cierto modo, artificiosas. A menudo se ha eludido el obstáculo neutralizando la diferencia [...] hubiera supuesto la imperdonable mutilación de una novela donde las formas del habla pertenecen al argumento de la obra. El vaivén de los registros verbales no es un mero recurso literario: es también un tema.

¿Qué hacer entonces? Entre los muchos lenguajes del castellano (todos felizmente locales) hemos escogido la versión cubana de la elocuencia caribeña. No, como es obvio, por afinidad lingüística o parentesco gramatical, sino por proximidad física y, sobre todo, psicológica (James 2016: 5).

A continuación, la nota explica que, primero, el novelista y traductor Javier Calvo se encargó de verter al castellano estándar el texto de James para que, después, la novelista Wendy Guerra cubanizara aquellos pasajes del original en los que se apreciaban los rasgos

7 Se puede leer un análisis minucioso de las traducciones al español de esta novela en Boyden, M. \& P. Goethals. 2011. “Translating the Watcher's Voice: Junot Díaz's The Brief Wondrous Life of Oscar Wao into Spanish". Meta 561: 20-41. 
del patois jamaicano mentados en el apartado 2.2. de este trabajo. De este modo, para traducir la variedad lingüística de la novela siguieron un método similar al de la versión de Obejas de La maravillosa vida breve de Oscar Wao. Optaron por un texto con marcas que transgreden la norma ortográfica de la lengua meta y por la estrategia denominada anatopismo o traducción dialectal paralela; es decir, convirtieron el dialecto del texto fuente en uno propio de la lengua meta de connotaciones similares, en este caso el español de Cuba. Al examinar los ejemplos siguientes, que se corresponden con las traducciones de los fragmentos de la novela incluidos en el apartado 2.2., también se aprecia que el texto en castellano recurre a la estrategia de compensación, es decir, algunas estructuras marcadas del texto fuente pasan a la lengua estándar en el texto meta y se marcan pasajes que no se desviaban de la norma en el original, de forma que las marcas dialectales no se corresponden una a una. Cabe mencionar, asimismo, que, salvo la nota sobre la traducción antes expuesta, la versión española de Breve historia de siete asesinatos no incluye notas del traductor ni otro tipo de paratexto con reflexiones acerca de la actividad traductora.

En el siguiente fragmento, narrado por Papa-Lo, el líder de la banda de delincuentes Storm Posse, se ve cómo la traducción al castellano opta por un texto con marcas que transgreden la norma ortográfica de la lengua meta, caso de los participios que terminan en "-ao" ("avisao", "cansao"), la transcripción de la pronunciación cubana mediante el eye-dialect ("veldá") y el empleo de calcos léxicos del inglés propios del español caribeño, como "men" para referirse de forma coloquial a un hombre en lugar del peninsular "tío":

Yo le avisé, ¿ok, excelencias? Hace tiempo que yo le venía avisando de que había gente cercana a él, amigos y enemigos, que lo iban a meter en candela. Todos conocemos al menos a uno, ¿veldá? Son el tipo de gente que no cambia, ¿no? Tienes muchas cosas en la cabeza pero nunca se les ocurre una sola idea. Siempre se están confabulando pero nunca tienen un plan. Eso es un tipo de persona. Ahí estaba mi amigo, la superestrella más grande del mundo, y el men seguía sin tener ni idea de cómo lograr salir del gueto quedando a buenas con todos. No quise decirle nombres, pero yo tenía avisao al Cantante. Le decía: tienes gente cerca que no va a cansarse hasta que te hunda, ¿me oíste? Estaba cansao de decírselo. Cansaísimo. Pero el men me soltaba esa risa suya, esa risa que se tragaba la habitación entera. Esa risa que sonaba como si ya tuviera su plan (James 2016: 40).

A continuación, se retoman los pasajes que ilustran la habilidad de Josey Wales para desenvolverse en varios registros. Mientras que su narración en primera persona se corresponde con el español estándar salpicado de vocablos propios de Centroamérica ("chueco", es decir, malo o defectuoso), luego se hace el tonto con su interlocutor, baja el registro y emplea apócopes como "to". Sin embargo, en el primer fragmento de la traducción se pierde el pasaje en el que Wales demuestra que sabe español y entiende lo que dice su interlocutor hispano (James 2015: 44), mientras que en el segundo fragmento del texto meta se observa la inclusión de una glosa intertextual (“y añadió en español”) que no está presente en el texto fuente para introducir una frase en castellano, marcada con tipografía cursiva para diferenciarla de las partes del diálogo que el interlocutor presupone que sí entiende Wales, en inglés en el original: 
No le dije que estaba a punto de poner a un hombre en Miami y a otro en Nueva York. No le dije que sabía el suficiente español para darme cuenta de que el señor era el hazmerreír de Latinoamérica. Hablé con él en un inglés chueco, como si fuera un negro ignorante, y le hice preguntas idiotas tipo: ¿Y en América to el mundo tiene pistola? ¿Qué clase de bala disparan en América? ¿Por qué no trasladan a Harry el Sucio a la delegación jamaicana? ¡Je, je, je! (James 2016: 65-66).

- ¡Ah! Disculpa, error mío - y añadió en español-: Pero todo es un error en este país de mierda.

- Ni la mitad de error que en la cagada de país del que vienes tú.

-Oye, ¿hablas español?

Asentí con la cabeza.

- ¿Y crees que lo sabe el hombre de la CIA?

Negué con la cabeza (James 2016: 205).

De manera análoga, el malentendido que ocasionan los referentes culturales que menciona Pierce, así como el contraste entre el inglés estadounidense del periodista y el jamaicano de los guardias de seguridad que vigilan la casa de Marley, se han compensado en la versión de Calvo y Guerra. Mientras que la comicidad de la confusión del título de la revista con el nombre del grupo de Mick Jagger y Keith Rcihards se mantiene en ambas lenguas, el malentendido que origina la mención a Led Zepellin se compensa y, en el texto meta, los guardias confunden el nombre del grupo de música con las "papelinas" de las que creen que habla el periodista, en contraste con "las lesbianas" a las que pensaban que se refería en la versión original (James 2015: 50). En la traducción no se compensan, no obstante, algunas de las marcas del inglés jamaicano del texto fuente, como los usos de los pronombres personales ("me no repent"), la falta de flexión en los verbos ("You never talk to me on no pone") o la pronunciación no estándar y burlesca a la que apunta el eye dialect ("sexetary"), que se intentan compensar con formas apocopadas ("na"):

- Me cago en la mierda. Pero mira esto, Taffie, Jesucristo ha resucitado.

- ¿Cómo? Pero si todavía no me he arrepentido de mis pecados.

El blanco no parece captar la broma. Yo me he apartado del medio, quizá con demasiado aspaviento.

- ¿Qué pasa, compadre? Alex Pierce, de Rolling Stone.

-Quieto, pare, Jesucristo de los jeans de pitillo. ¿Ya sabe Jehová que vas contando mentiras? Ya han venido dos tipos de Rolling Stone, uno se llamaba Keith y el otro Mick, y ninguno se parece a ti.

- Pero todos se parecen, Eddie.

- Es verdad. Es verdad.

- Yo soy de la revista Rolling Stone. Hemos hablado por teléfono.

- Conmigo no has hablado por teléfono.

- Bueno, con alguien de la oficina. Con su secretaria o alguien, no sé. Soy de la revista... De Estados Unidos... Cubrimos todos los eventos, desde Led Zeppelin a Elton John. No entiendo nada, la secretaria me dijo que viniera el 3 de diciembre a las seis de la tarde cuando él estuviera en el descanso de los ensayos, y aquí estoy.

-Jefe, no sé na de ninguna secretaria.

- Pero...

- Mira, tenemos órdenes estrictas. Ni entra ni sale nadie más que la familia y la banda. 
- ¡Vaya! ¿Y por qué todo el mundo lleva un arma automática? ¿Sois de la policía? No os parecéis en nada a los guardias de seguridad de la última vez que vine.

-Eso a ti te importa una mierda. Mejor te largas.

-Eddie, ¿todavía te está molestando el tipo de la verja?

-Dice que es de una revista que habla de papelinas y de Elton John.

-No, de Led Zeppelin y de...

-Dile que se largue.

— ¿Por qué dejan que los ayude? (James 2016: 72).

En cuanto al último fragmento seleccionado de la novela, las características del inglés jamaicano del policía que detiene a Demus, de las que se habla en la sección 2.2. de este trabajo, se compensan por medio de contracciones ("p'adelante", "p'al suelo"), apócopes ("to", "tos"), participios que terminan en "-ao" ("singao"), frases hechas coloquiales ("le colgaran a alguno el muerto") que acentúan el tono vulgar de la edición española y por la transcripción de la pronunciación caribeña mediante el eye-dialect (“singuen"):

Los policías, y conté seis, nos dijeron: uno de ustedes es un violador asqueroso que viola a mujeres decentes cuando vuelven de alabar al Señor. Y como son tos unos mentirosos de mierda, ni siquiera le voy a pedir al culpable que dé un paso p'alante. Y nosotros no sabíamos qué hacer porque como le colgaran a alguno el muerto de la violación, ni siquiera iba a llegar a la cárcel porque la policía le iba a pegar un tiro antes. Así que el primer policía, que era el que hablaba todo el tiempo, dijo: pero sí sabemos cómo atraparte. ¡Tos p'al suelo ahora mismo, delen! Nosotros no lo creíamos, así que nos miramos los unos a los otros y yo miré las burbujas de jabón, que estaban reventando una a una y dejándome las vergüenzas al aire. El policía pegó dos tiros al aire y dijo: ; to el mundo pa'l suelo ya! Así que nos tiramos pa'l suelo [...] Y ahora oigan lo que quiero que hagan. Quiero que todos se singuen el suelo bien singao [...] ¡He dicho que se singuen el suelo! (James 2016: 76).

Las críticas de la traducción al español de Breve historia de siete asesinatos elogian el trabajo de Calvo y Guerra, pues reconocen que la mezcla de dialectos del texto fuente supone un problema de difícil solución a la hora de verter la novela al castellano y señalan la necesidad de jugar con la norma lingüística para que no se pierda la polifonía que caracteriza la obra de James (Pardo 2016). Además, destacan el empeño de los traductores por reproducir la oralidad de novela, que describen como "un viaje por un archivo de historia oral de la Jamaica de los setenta y ochenta" (Sancho Cardiel 2016).

\section{CONCLUSIONES}

Al comienzo de este estudio se ha planteado el escollo de la traducción al castellano de la variedad lingüística presente en la novela Breve historia de siete asesinatos, de Marlon James. Dado que en esta obra coral se alternan las voces de quince narradores y se reflejan las particularidades del criollo jamaicano que hablan varios de ellos por medio de alteraciones de la ortografía estándar y la técnica denominada eye-dialect, primero se han analizado las distintas estrategias con las que cuenta el traductor para hacer frente a este problema. Son varios los traductólogos que manifiestan la necesidad de reflejar estas desviaciones de la norma lingüística en la lengua meta (López García 1991: 93; Rabadán 1991: 79-109; 
Hatim y Mason 1995: 55-64) y, con este fin, se han considerado las propuestas de Tello Fons (2012: 137-158) de optar por un texto con marcas en la lengua de llegada, que transgreda la norma, o por uno estandarizado; además de las seis estrategias para la traducción del dialecto literario que recopilan Rica Peromingo y Braga Riera (2015: 133-142). No obstante, también se ha señalado, en el apartado 3.2., la tendencia a la neutralización de las variedades lingüísticas por criterios editoriales; si bien se han apuntado las notables excepciones de las traducciones de Llámalo sueño y La maravillosa vida breve de Óscar Wao de Sáenz y Obejas, respectivamente.

Tras estas consideraciones previas, se ha observado qué estrategias se emplearon para traducir al castellano el dialecto jamaicano de Breve historia de siete asesinatos. La nota sobre la traducción que abre la novela aborda este mismo problema y cuenta que la escritora Wendy Guerra se encargó de cubanizar aquellos pasajes escritos en patois después de que Calvo los vertiera al español estándar. De este modo, y como ilustran los ejemplos del apartado 4., se observan las estrategias de traducción dialectal paralela o anatopismo, es decir, la correspondencia con un dialecto concreto de la lengua meta (el español cubano en este caso). Asimismo, los traductores se valieron de la compensación, de forma que las marcas dialectales no se corresponden una a una con las del original. El resultado es un texto meta que transgrede la norma lingüística por medio de apócopes, contracciones, frases hechas coloquiales y transcripciones de la pronunciación no estándar de los protagonistas de esta novela coral. En futuras investigaciones se podrían contrastar estas estrategias para la traducción al castellano de la variedad lingüística de Breve historia de siete asesinatos con las que se emplearon en la mentada versión catalana de Moton i Lara.

\section{REFERENCIAS}

Bailey, B. L. 1966. Jamaican Creole Syntax: A Transformational Approach. Cambridge: Cambridge University Press.

Bakhtin, M. M. 1981. The Dialogic Imagination: Four essays. Trad. C. EMERSON y M. HOLQUIST. Austin: University of Texas Press.

BERnÁRdEZ SANChís, E. 2012. “Traduttore-traditore... ¿o editore-destruttore?” Lengua, traducción, recepción: en honor de Julio César Santoyo. Eds. R. RABADÁN, T. GUZMÁN y M. FERNÁNDEZ. León: Universidad de León. 93-115.

Blincoe, N. 2015. “A Brief History of Seven Killings by Marlon James, Review: Vivid and Powerful.” The Telegraph. 9 Mar. 2017. http://www.telegraph.co.uk/culture/books/ bookreviews/11213049/A-Brief-History-of-Seven-Killings-by-Marlon-James.html

CALvo, J. 2016. El fantasma en el libro. Barcelona: Seix Barral.

CRYstal, D. y Ivić, P. 2010. “Dialect.” Encyclopaedia Britannica. 8 Mar. 2017. https:// global.britannica.com/topic/dialect

D’Amore, A. M. 2010. “Traducción en la zona de contacto.” Mutatis Mutandis 3, 1: 30-44.

DíAz, J. 2007a. The Brief Wondrous Life of Oscar Wao. Nueva York: Riverhead Books. 
---- 2007b. La maravillosa vida breve de Óscar Wao. Trad. A. OBEJAS. Barcelona: Mondadori.

Gibson, K. 1988. "The Habitual Category on Guyanese and Jamaican Creoles." American Speech 63, 3: 195-202.

Harvey, C. 2015. "Marlon James interview: 'I didn’t want to fall into a pornography of violence'." The Telegraph. 9 Mar. 2017. http://www.telegraph.co.uk/culture/ books/11672011/Booker-Prize-author-Marlon-James-interview.html

Hatim, B. y Mason, I. 1995. Teoría de la traducción: Una aproximación al discurso. Trad. S. PEÑA. Barcelona: Ariel.

James, M. 2015. A Brief History of Seven Killings. Londres: Oneworld.

----. 2016. Breve historia de siete asesinatos. Trad. J. CALVO y W. GUERRA. Barcelona: Malpaso.

JimÉnez CARRA, N. 2011. "La traducción del cambio de código inglés-español en la obra The Brief Wondrous Life of Oscar Wao, de Junot Díaz.” Sendebar 22: 159-180.

Julià BALlBè, J. 1997. “The Adventures of Huckleberry Finn i les traduccions impossible.” Traducció i literatura: Homenatge a Ángel Crespo. Eds. S. GONZÁLEZ RÓDENAS y F. LAFARGA. Vic: Eumo. 195-202.

LANDERs, C. E. 1999. Literary Translation: A Practical Guide. Clevedon / Buffalo / Toronto: Multilingual Matters.

LAZAR, Z. 2014. “A Brief History of Seven Killings, by Marlon James.” The New York Times. 9 Mar. 2017. https://www.nytimes.com/2014/10/26/books/review/a-brief-history-ofseven-killings-by-marlon-james-review.html

López García, D. 1991. Sobre la imposibilidad de la traducción. Cuenca: Universidad de Castilla-La Mancha.

MÄÄттё, S. K. 2004. "Dialect and point of view: The ideology of translation in The Sound and the Fury in French." Target 16, 2: 319-339.

Obejas, A. 2012. "Translating Junot.” Chicago Tribune. 9 Mar. 2017. http://www. chicagotribune.com/lifestyles/books/ct-prj-0916-book-of-the-month-20120914-story. html

Paradela LóPez, D. 2014. “Traducir dialectos.” El Trujamán: Revista diaria de traducción. 9 Mar. 2017. http://cvc.cervantes.es/trujaman/busqueda/resultadosbusqueda. asp?Ver=50\&Pagina $=1 \&$ Titulo=Traducir\%20dialectos $\&$ OrdenResultados $=2$

PARdo, P. 2016. "Bob Marley: una novela de violencia.” El Mundo. 9 Mar. 2017. http:// www.elmundo.es/cultura/2016/04/02/57002b77268e3e00738b4639.html

RABADÁN, R. 1991. Equivalencia y traducción: Problemática de la equivalencia translémica inglés-español. León: Universidad de León.

Rica Peromingo, J. P. y Braga Riera, J. 2015. Herramientas y técnicas para la traducción inglés-español: los textos literarios. Madrid: Escolar y Mayo. 
Rотн, H. 1990. Llámalo sueño. Trad. M. SÁENZ. Madrid: Alfaguara.

SÁEnZ, M. 2000. "Dialectos dilectos.” El Trujamán: Revista diaria de traducción. 8 Mar. 2017. http://cvc.cervantes.es/trujaman/anteriores/noviembre_00/03112000.htm

SÁNChez Galvis, J. 2013. "Una lectura dialectal de la historia de la traducción." MonTI: Monografías de traducción e interpretación 5: 139-164.

SANCHO CARDIEL, M. 2016. "Retratar la violencia requiere mucho autocontrol." El País.9 Mar. 2017. http://cultura.elpais.com/cultura/2016/06/27/actualidad/1467013449_710010. html

Tello Fons, I. 2012. “Traducción de la variación lingüística: Una visión diacrónica.” Hikma 11: 133-159.

Twain, M. 2001. The Adventures of Tom Sawyer. The Adventures of Huckleberry Finn. Ware: Wordsworth Classics.

Walpole, J. R. 1974. "Eye Dialect in Fictional Dialogue." College Composition and Communication 25, 2: 191-196.

Zanger, J. 1966. "Literary Dialect and Social Change." Midcontinent American Studies Journal 7, 2: 40-48. 